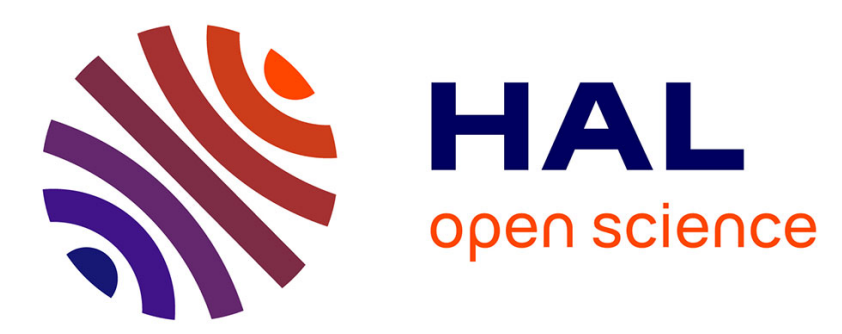

\title{
Logical handling of uncertain, ontology-based, spatial information
}

Florence Dupin de Saint Cyr - Bannay, Henri Prade

\section{To cite this version:}

Florence Dupin de Saint Cyr - Bannay, Henri Prade. Logical handling of uncertain, ontology-based, spatial information. Fuzzy Sets and Systems, 2008, 159 (12), pp.1515-1534. 10.1016/j.fss.2008.01.010 . hal-03324617

\section{HAL Id: hal-03324617 \\ https://hal.science/hal-03324617}

Submitted on 23 Aug 2021

HAL is a multi-disciplinary open access archive for the deposit and dissemination of scientific research documents, whether they are published or not. The documents may come from teaching and research institutions in France or abroad, or from public or private research centers.
L'archive ouverte pluridisciplinaire HAL, est destinée au dépôt et à la diffusion de documents scientifiques de niveau recherche, publiés ou non, émanant des établissements d'enseignement et de recherche français ou étrangers, des laboratoires publics ou privés. 


\title{
Logical handling of uncertain, ontology-based, spatial information
}

\author{
Florence Dupin de Saint-Cyr Henri Prade \\ Institut de Recherche en Informatique de Toulouse, \\ Université Paul Sabatier, \\ 31062 Toulouse Cedex 09, France.
}

\begin{abstract}
The paper describes a logical framework for handling uncertain spatial information, and merging it when it comes from multiple sources. For this purpose, we use a simple logical formalization for spatial ontologies and for property ontologies relative to different universes of discourse (these ontologies only involve subsumption and mutual exclusiveness relations), since spatial information typically associates properties to sets of parcels that are themselves described in terms of the spatial and/or property vocabularies appearing in the ontologies.

Apart from the ontological information describing relations between labels, we propose to represent a piece of spatial information as a pair, called "attributive formula", associating a property formula to a set of parcels (represented by a spatial formula). A set of inference rules is given in order to be able to reason from these attributive pairs. Then, we examine how uncertainty can be encoded in attributive information, using possibilistic logic in a reified manner with respect to parcels. Another important issue pointed out in this paper is that there are two ways to link a property to an area, a first meaning is that the property is true everywhere in the area, a second meaning is that the property is at least true somewhere in the area. This distinction is necessary in order to be able to use both ontological information (which can be encoded by "everywhere" attributive-formulas) and attributive information (which contain the two kinds of attributive-formulas).

Lastly, the paper studies how information fusion problems can be handled in the context of spatial data. The problems encountered do not come only from the uncertainty and the possible inconsistency of information as in any information fusion situations, but also from the fact that sources may use different space partitions and may not explicitly specify the somewhere or everywhere reading associated to the information.
\end{abstract}

Key words: spatial information, ontologies, uncertainty, possibilistic logic, fusion.

Email addresses: 


\section{Introduction}

The management of multiple source, symbolic or numerical, information raises different types of representation, reasoning, and fusion problems due to the uncertainty and the heterogeneity of information [7]. The spatial distribution of information in geographical information systems $[2,19,27]$ adds new features to these problems. Spatial information may involve numerical as well as symbolic attributes whose evaluation may use different vocabularies according to the sources. The sources may also use different space partitions. Moreover, there may exist possible dependencies between the pieces of information pertaining to parcels that are close for instance. Spatial information fusion issues may more generally take place in a variety of information handling problems such as the management of multiple source spatial databases systems, the prediction or the detection of changes over time of land-cover parameters (e.g., [32]), or in best location decision problems using multiple information sources and multiple criteria.

Dealing with spatial data requires relatively powerful representation languages. Geographic representation may privilege object-based or location-based views; see, e.g. [22] for a discussion. Besides, a need is the ability to express structured information about the vocabulary used. Ontologies are often used for representing this kind of information [19]. The fusion of ontology-based geospatial information has been already considered, generally focusing on the problem raised by the heterogeneity of vocabularies used by different sources. In particular, Duckham and Worboys [17] has proposed a method for fusing ontologies on the basis of the instance-level information given by each source and projected on a product partition of the partitions used by the sources. It results in an integrated taxonomy lattice where some new labels can be built as the meet of labels of the different ontologies when necessary. The handling of uncertain instance-level information is also discussed in this setting. In the geographical context, fusion may also focus on more specific problems such as data matching on the basis of distances and similarity between toponyms, see, e.g. [24] for an approach using Shafer theory of evidence. In this paper, we are not dealing with ontology integration, nor with data matching, but rather, we discuss the problems raised by the merging of information provided by different sources using different space partitions, and expressed with more or less precise labels belonging to the same (possibly integrated) ontology.

There exist very few authors using a formal logic approach for handling geographical information, up to some noticeable exceptions such as Papini et al. [32]. In the following, we also use a logical framework for processing ontological information and "attributive formulas" linking a set of parcels to a

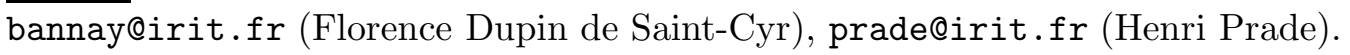


property statement. The simple type of ontology that we consider can be logically expressed by three and only three kinds of information about vocabulary symbols, namely, 1) a label can be a sub-category of another label, 2) a label is the reunion of its sub-labels, 3) labels referring to the most specific classes are mutually exclusive two by two.

Another need when dealing with spatial information is to be able to take into account any spatial information even if it is imprecise or pervaded with uncertainty [21]. This is why we allow for "uncertain attributive formulas" linking a set of parcels to a property associated with a certainty degree. An (uncertain) attributive formula allows us to express that for any parcel of a given set of parcels, (we are sure at least at a given level that) a property is true. We also provide the possibility to express that at least one parcel in a set of parcels has a given property. Moreover it is also useful to distinguish between statements that hold everywhere on set of parcels, or maybe only somewhere in a parcel.

A very preliminary version [10] of this paper focused on an informal discussion of the problems raised by the handling of uncertain spatial information possibly referring to several property ontologies. The present paper makes a step forward by providing a logical framework for handling spatial information and ontological information. Another step is made by handling the merging of spatial information in the general setting of logical information fusion.

In such a spatial data representational language, even if ontological information and attributive formulas can be expressed in the same formalism, it is important to distinguishing cautiously their "somewhere" or "everywhere" readings when processing them. Indeed, we show that while inheritance relations coming from an ontology can safely be integrated to a spatial knowledge base expressed by attributive formulas, terminological mutual exclusiveness relations between labels cannot. Their integration is possible if their precise reading (everywhere, or somewhere) is explicited or if a strong hypothesis is made, namely that an elementary parcel cannot satisfy two terminologically mutually exclusive properties (hence, e.g., forbidding a parcel to contain both "Orchards" and "Cereals").

The paper is organized in the following way. Section 2 discusses issues related to the representational needs in the spatial information domain. In section 3 , we describe a simple way to logically represent ontological information (in our restricted sense). In the fourth section we introduce the notion of an "attributive formula" as a reified formula where a property is attached to a set of parcels, with the meaning that each parcel has this property. Then "uncertain attributive formulas" are defined, and finally, the explicit precision of the "somewhere" or "everywhere" reading associated to an attributive formula is introduced. Section 5 first recalls the basis of information fusion in a possi- 
bilistic setting, and then shows how to integrate its principles in the context of "attributive formulas" representing spatial information. It is illustrated on a spatial information fusion example, inspired from [28]. Section 6 discusses the problem of reformulating spatial information in terms suitable for a user, and then briefly introduce two potential extensions of the representation language. The first extension allows us to represent the fact that a property holds on a given percentage of a parcel. The second extension would allow us to represent "positive" information stating what is known as being "guaranteed possible", together with classical ("negative") information that states what is impossible or excluded, when dealing with imprecision or uncertainty.

\section{The representational needs}

A specific aspect of spatial information is that information is associated to parcels that are geographically identified (the parcels are usually defined through partitions of the territory). This means that information is of the type "propertyobject", where the objects are here parcels or sets of parcels. Moreover, information may be numerically or symbolically expressed. It is often the case that the symbolic labels used are referring to a hierarchy of concepts rather than belonging to an unstructured set. Lastly, both numerical and symbolic information may be pervaded by several forms of uncertainty and imprecision.

\subsection{Ontology of properties}

As just said, the attribute values or properties associated to parcels may be not just elements of an attribute domain, but may be symbolic labels taken from a vocabulary organized in an ontology. We use the term ontology here, in the weak technical sense of a graph structure between concepts, where the arrows encode specialization/subsumption relations. For instance, Figure 1 provides an example of (a part of) an ontology about land cover (where arrows refer to generalization relations).

Observe that an ontology may neither have, in general, a tree structure (connected and with no simple cycle), nor a lattice structure (which requires that each pair of nodes has a unique least upper bound and a unique greatest lower bound). The ontology of Figure 1 is not a lattice since, for instance, there is no greatest lower bound of \{Woods, Agricultural areas\}, indeed there are two elements, namely "Ornamental trees" and "Orchards" which are maximal among the labels that are lower than "Woods" and "Agricultural areas", but none of them is greater than the other. 


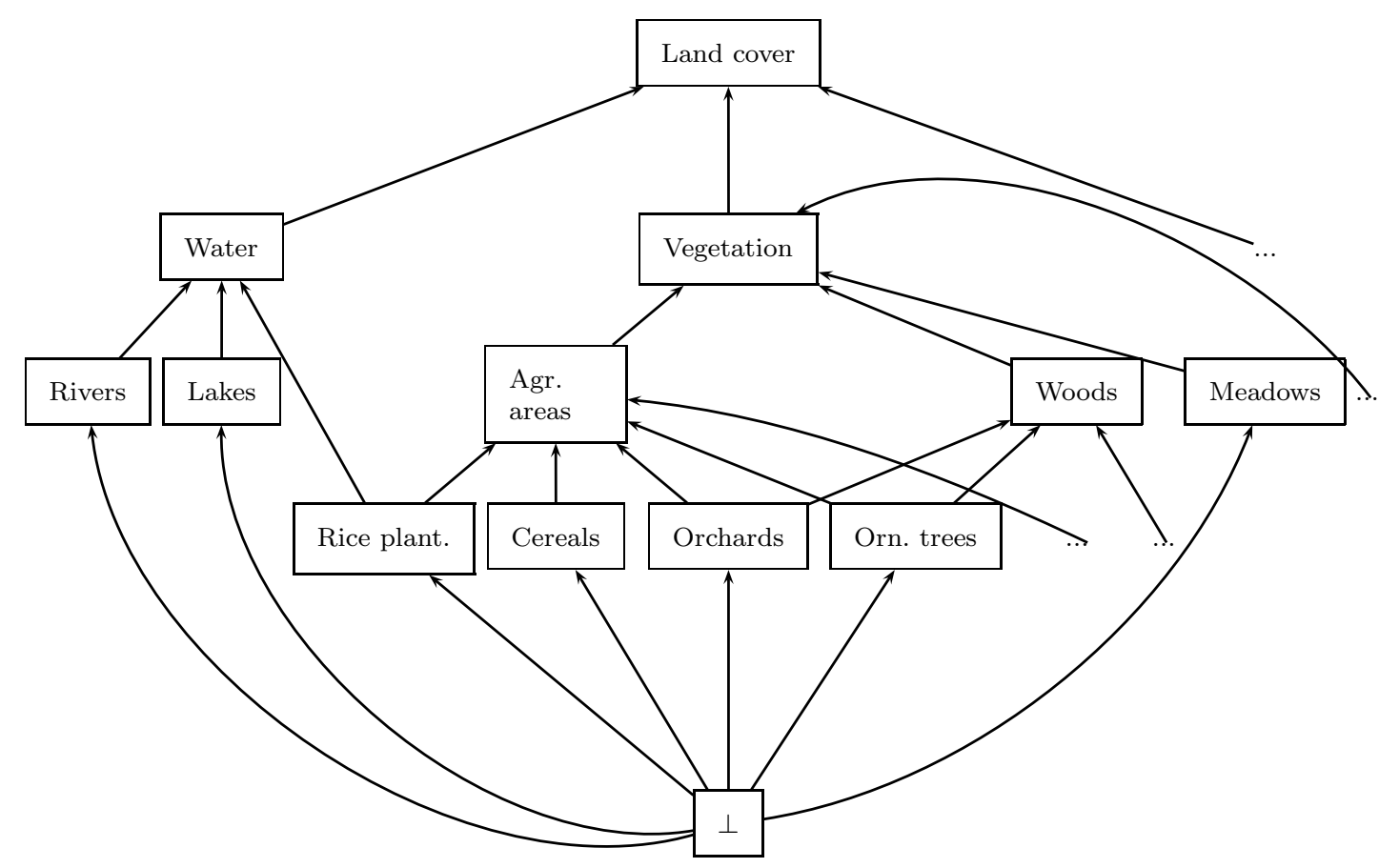

Fig. 1. Example of a fragment of an ontology

\subsection{Ontology of parcels}

In the partition of a territory, particular subsets of parcels may have names. The inclusion relation between sets of parcels can be also represented by an ontology, as for properties. However, one may further assume here that, even if several spatial ontologies may coexist (due to the existence of several points of view in the way the geographical space can be portioned in a meaningful way), all these ontologies share the same set of elementary parcels. See Figure 2 that exhibits two spatial ontologies $G s$ and $G s^{\prime}$, where in each case, the leaves, i. e. our elementary parcels, are the parcels $p_{1}, \ldots, p_{6}$. A similar assumption seems more difficult to do for "property" ontologies, used by different sources.

\subsection{Uncertainty in spatial information}

In the field of spatial information, as in other fields, information may be imprecise, pervaded with uncertainty or be inconsistent. Spatial information may take different classical formats.

- In a relational data base style, each object, i.e., a parcel here, is described in terms of attributes, and is thus associated with a set of attribute values.

- More simply in formal concept analysis [31,20], a relation specifies the links between objects and properties. This enables us to describe sets of objects both in extension and in intention, through a set of characteristic prop- 


\begin{tabular}{|l|l|}
\multicolumn{1}{c}{$G s$} \\
\hline$p_{1}$ & $p_{23}$ \\
\hline$p_{4}$ & $p_{56}$ \\
\hline
\end{tabular}

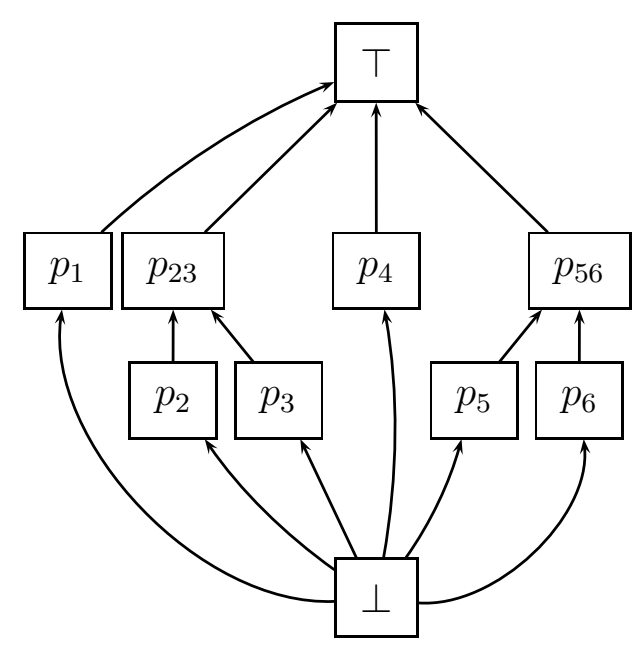

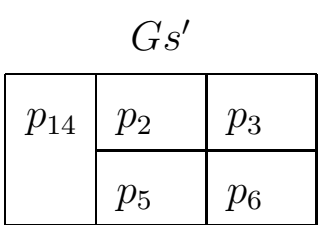

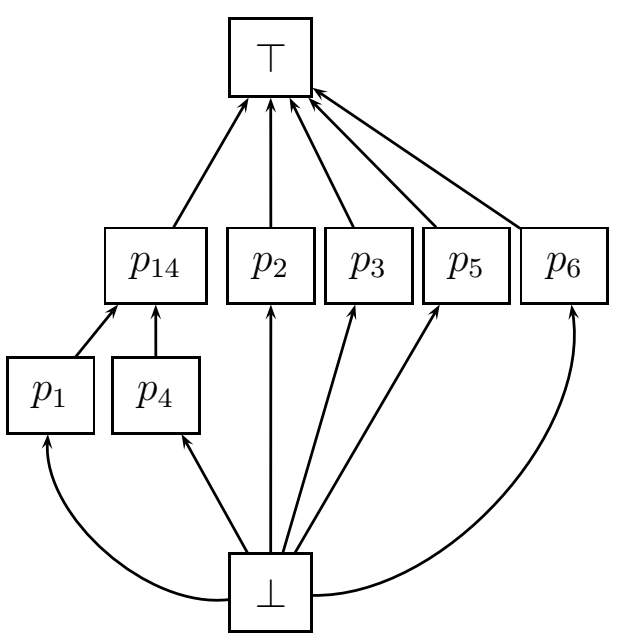

Fig. 2. Two space ontologies

erties. Then a pair (extension, intention) whose components are referring to each other in a bi-univoque way formally defines a concept. However, the vocabulary is often insufficient for describing any subset of objects in a non-ambiguous way, or conversely given a set of properties there may be no proper set of objects that satisfy them and only them.

- The two previous types of representation can be encoded using fragments of first order logic, where constants can either represent objects or attribute values.

When uncertainty takes place, attribute values of objects may become illknown, and then represented by distributions over possible values or over logical interpretations. In the case of relational database representations, the distributions are defined on the attribute domains, in formal concept analysis the distributions are defined on a yes-no domain referring to the fact that the object has, or not, the property. In the logical formalism, the formulas become associated to certainty levels that act as constraints on underlying distributions over interpretations.

For instance, one may know that a parcel is covered either by "cereals" or by "meadows", where the two categories are mutually exclusive. Such a disjunctive value is not allowed in standard relational databases, or in standard formal concept analysis, while it raises no problem in a logical representation. Moreover, in case of imprecise information (as in the above example), it might be useful to represent that some alternatives are more possible or likely than others. Disjunction may apply also to non mutually exclusive categories, as 
in the expression "cereals or vegetation" whose intended meaning could be as such "it is likely to be cereals, although any other vegetation might be possible".

Since we are dealing with spatial information, there may be some ambiguity on the way attribute values or properties apply to the parcels: they may apply either to the whole parcel or only to a sub-part of it. For instance, if it is known that a parcel is covered by "rocks" and "sand" then it may mean that a part of the parcel is covered by "rocks" and another part by "sand". In such a case it may be interesting to make the representation more precise by associating to the logical conjunction of the categories some additional information about the percentage of the parcel respectively covered by "rocks" or by "sand".

\section{A logical encoding of taxonomic information}

Let $\mathscr{L}$ be a propositional logical language built on a vocabulary $\mathscr{V}$ where the usual propositional connectives are denoted by $\wedge, \vee, \rightarrow$ (representing the logical "and", "or", and material implication respectively). In the following, we introduce the logical encoding that we associate to an ontology.

Although description or terminological logics [1] have been developed, as tractable fragments of first order logics, for encoding ontologies, we use a simplified propositional encoding of the intuitive notion of ontology [30] in this paper, which is sufficient for handling fusion problems of symbolic information expressed by means of category labels.

Definition 1 An ontology is a graph $G=(X, U)$ where $X \subseteq \mathscr{L}$ is a set of formulas (each of these formulas encodes a category referred to in the ontology) and $U$ is a set of directed edges or arcs. Each arc $(\varphi, \psi) \in U$ represents the fact that $\varphi$ is a subclass of $\psi$. An ontology is connected, without circuit and it admits one and only one source (vertex without predecessor) equal to $\perp$ and one and only one sink (vertex without successor). Moreover all the subclasses that have $\perp$ as predecessor are mutually exclusive two by two. These subclasses are called leaves. Each class which is not a leave nor $\perp$ is equivalent to the union of all its predecessors.

The levels of the ontology are defined inductively as: Level $0\left(L_{0}\right)$ is the set of vertices that have no predecessor, Level $i$ is the set of vertices that have no predecessor in $G \backslash\left(L_{0} \cup \ldots L_{i-1}\right)$ and so on.

Example 1 The ontology of Fig.1 have four levels: 
$L_{0}: \perp$

$L_{1}$ : Rivers; Lakes; Rice plantations; Cereals; Orchards; Orn. trees;Meadows

$L_{2}$ : Water; Agricultural areas; Woods

$L_{3}$ : Vegetation

$L_{4}:$ Land cover

Let $\Gamma^{+}(x)$ and $\Gamma^{-}(x)$ be the set of successors of $x$ and the set of predecessors of $x$ respectively. An ontology will be encoded in the following way.

Definition 2 (logical encoding of an ontology) Any graph $G=(X, U)$ representing an ontology can be associated to a set $L_{G}$ of formulas that hold:

1. $\forall(\varphi, \psi) \in U$,

2. $\forall \varphi \in X \backslash\left\{L_{1} \cup L_{0}\right\}$,

3. $\forall \varphi, \psi \in L_{1}$,

4. $\forall(\varphi, \psi) \in X \times X$, s.t. $\varphi \vdash \psi$, it exists a directed path from $\varphi$ to $\psi$ in $G$. it holds that $\varphi \rightarrow \psi$.

it holds that $\varphi \rightarrow \bigvee_{\varphi_{i} \in \Gamma^{-}(\varphi)} \varphi_{i}$.

it holds that $\varphi \wedge \psi \rightarrow \perp$.

The first formula expresses that an inclusion relation holds between two categories. The second is a kind of categorical closed world assumption expressing that all the sub-categories of a category appear in the ontology. It is always possible to satisfy this assumption by adding for each category $C$ a sub-category "other elements of C". The third formula expresses that at the bottom of the hierarchy, sub-categories are mutually exclusive two by two. It is usually assumed in an ontology that all the inclusion relations are known, namely, in other words, if there is no directed path between two categories then there cannot exist an inclusion relation between them. This is expressed by the last requirement. It expresses that the graph must be complete with respect to its logical encoding, any implication that holds between two formulas of the ontology should be readable in the graph under the form of an arc, or more generally a path.

From this definition, it follows that $\forall \varphi \in X, \varphi \rightarrow \bigwedge_{\varphi_{i} \in \Gamma^{+}(\varphi)} \varphi_{i}$. It also follows that $\forall \varphi \in X, \varphi \rightarrow$ Sink where Sink is the sink of $G$ (since $G$ is connected, using Property 1 of Definition 2). Another consequence is that the simplest non-trivial ontology contains at least two nodes different from $\perp$ and Sink. These two nodes are mutually exclusive.

Proposition 1 Given any pair of formulas $(\varphi, \psi) \in X \times X$, the logical encoding of the ontology $G=(X, U)$ allows us to decide if

- $\{\varphi \wedge \psi\} \cup L_{G}$ is consistent or not;

- if $\varphi \cup L_{G} \vdash \psi$ or not; 
Example 2 See Figure 1, considering the formulas Water and Ornamental_trees, using the logical encoding $L_{G}$ of the ontology given in Figure 1, we get that $\{$ Water $\} \cup L_{G} \vdash$ Rivers $\vee$ Lake $\vee$ RicePlantations.

Moreover, $\{$ Ornamental_trees $\wedge$ Rivers $\} \cup L_{G} \vdash \perp$

and $\{$ Ornamental_trees $\wedge$ Lakes $\} \cup L_{G} \vdash \perp$,

hence $\{$ Water $\wedge$ Ornamental_trees $\} \cup L_{G} \vdash \perp$.

Similarly, we can establish that Water and Vegetation have a common subclass since $\{$ Water $\wedge$ Vegetation $\} \cup L_{G} \vdash$ Rice_plantation.

Hence, $L_{G} \vdash$ Water $\wedge$ Vegetation $\leftrightarrow$ Rice_plantation.

We have only illustrated our view of an ontology for property labels. However, Definition 2 and Proposition 1 are supposed to apply as well for spatial ontologies.

\section{A representation language for spatial information}

The logical representation of spatial information makes use of the following notations. Let $\mathscr{P}=\left\{p_{1}, \ldots, p_{n}\right\}$ be a set of symbolic labels attached to elementary parcels in the sense of a spatial ontology $G s$ encoded by $L_{G s}$ in a logical language $\mathscr{L}_{s}$ where the formulas of $\mathscr{L}_{s}$ are denoted by Roman letters. Thus these elementary parcels are supposed to be the leaves of the ontology $G s$. The third condition in the definition of ontologies guarantees their mutual exclusiveness. Besides, we shall consider a set of formulas expressed in a language $\mathscr{L}_{i}$, called $i$-formulas that are formulas based on a vocabulary $\mathscr{V}_{i}$, denoted by Greek letters. $\mathscr{V}_{i}$ and $\mathscr{V}_{s}$ may be the underlying vocabularies of ontologies of properties and parcels in the sense of the previous section, but this aspect will be left aside until section 6 .

\subsection{Attributive formulas}

The core of spatial information is attributive in nature. A piece of spatial information expresses a link between the description of a parcel and some property. This attributive link property-parcel should not be confused with two other basic links that we already encountered: i) link property-property (reflecting the knowledge encoded in an ontology $G i$ of properties, or ii) link parcel-parcel (given by an ontology Gs of parcels).

Since we need to express binary links, our representational language is built on ordered pairs of formulas of $\mathscr{L}_{i} \times \mathscr{L}_{s}$, here denoted $(\varphi, p)$. Such formulas should be understood as formulas of $\mathscr{L}_{i}$ reified by association with a set of parcels 
described by a formula of $\mathscr{L}_{s}$. In other words, to each formula is attached a set of parcels, where this formula applies. More precisely, $(\varphi, p)$ expresses that $\varphi$ is true for each elementary parcels satisfying $p$. Another understanding would view $(\varphi, p)$ as the material implication $\neg p \vee \varphi$ in the language based on the union of the two vocabularies $\mathscr{V}_{i}$ and $\mathscr{V}_{s}$. Alternatively, in a first order logic language view, this may be also understood as $\forall x, p(x) \rightarrow \varphi(x)$, here $p(x)$ means " $x \in p$ ", equating formula $p$ with the union of elementary parcels $x$ satisfying $p$. A pair $(\varphi, p)$ will be called an attributive formula.

Definition 3 (attributive formula) An attributive formula $f$, denoted by a pair $(\varphi, p)$, is a formula of the propositional language based on the vocabulary $\mathscr{V}_{i} \cup \mathscr{V}_{s}$ where the logical equivalence $f \equiv \neg p \vee \varphi$ holds and $p$ contains only variables of the vocabulary $\mathscr{V}_{s}\left(p \in \mathscr{L}_{s}\right)$ and $\varphi$ contains only variables of the vocabulary $\mathscr{V}_{i}\left(\varphi \in \mathscr{L}_{i}\right)$

The intuitive meaning of $f=(\varphi, p)$ is that for the set of elementary parcels that satisfy $p$, the formula $\varphi$ is true.

Observe that there exist propositional formulas built on the vocabulary $\mathscr{V}_{i} \cup \mathscr{V}_{s}$ which cannot be put under the attributive form, e.g., $a \wedge p_{1}$ where $a$ is a literal of $\mathscr{V}_{i}$ and $p_{1}$ a literal of $\mathscr{V}_{s}$; see also Section 6.1 for further discussions.

The introduction of classical connectives $(\wedge, \vee$ and $\neg)$ between attributive formulas does make sense, since any pair $(\varphi, p)$ is a classical formula. Thus, formulas such as $\neg(\varphi, p)$ or $\left(\varphi_{1}, p_{1}\right) \vee\left(\varphi_{2}, p_{2}\right)$ are allowed.

From the above definition of $(\varphi, p)$ as being equivalent to $\neg p \vee \varphi$, several inference rules straightforwardly follow from classical logic:

Proposition 2 (inference rules on attributive formulas)

1. $\left(\neg \varphi \vee \varphi^{\prime}, p\right),\left(\varphi \vee \varphi^{\prime \prime}, p^{\prime}\right) \vdash\left(\varphi^{\prime} \vee \varphi^{\prime \prime}, p \wedge p^{\prime}\right)$

2. $(\varphi, p),\left(\varphi^{\prime}, p\right) \vdash\left(\varphi \wedge \varphi^{\prime}, p\right)$

3. $(\varphi, p),\left(\varphi, p^{\prime}\right) \vdash\left(\varphi, p \vee p^{\prime}\right)$

4. if $p^{\prime} \vdash p$ then $(\varphi, p) \vdash\left(\varphi, p^{\prime}\right)$

5. if $\varphi \vdash \varphi^{\prime}$ then $(\varphi, p) \vdash\left(\varphi^{\prime}, p\right)$

Corollary 1 The converse of 2. holds: $\left(\varphi \wedge \varphi^{\prime}, p\right) \vdash(\varphi, p),\left(\varphi^{\prime}, p\right)$

Proof : This property is due to 5.

Corollary $2(\varphi, p),\left(\psi, p^{\prime}\right) \vdash\left(\varphi \vee \psi, p \vee p^{\prime}\right)$

Proof : Due to 5. and 3. 
This corollary allows to deduce the following cutting rule:

$$
(\varphi, p),(\neg \psi, \neg p) \vdash(\psi \rightarrow \varphi, \top)
$$

Note that $(\psi \rightarrow \varphi, \top)$ means here that for each parcel, the implication $\psi \rightarrow \varphi$ holds.

Example 3 For instance, let us reason on a set of parcels representing countries in a given area $A$. If we know that every Eastern country in $A$ has a despotic government (despot,east) and if we know that in $A$ there is no oil exploitation outside Eastern countries ( $\neg$ oil, ᄀeast) then we could conclude that oil exploitations lead to despotism in $A$ (oil $\rightarrow$ despot, $\top$ ), meaning that this implication is true in each country (of $A$ ).

Corollary $3(\varphi, p),\left(\psi, p^{\prime}\right) \vdash\left(\varphi \wedge \psi, p \wedge p^{\prime}\right)$

Proof : Due to 2. and 4.

Example 4 Let us consider the two facts $\left(\right.$ Cereals, $\left.p_{1}\right)$ and (Orchards, $p_{1} \vee$ $p_{2}$ ) where $p_{1}$ and $p_{2}$ are two elementary parcels and Cereals and Orchards are two literals of $\mathscr{V}_{i}$. Note that (Orchards, $p_{1} \vee p_{2}$ ) means that Orchards is true for $p_{1}$ and for $p_{2}$, at least. In particular, the second formula entails $\left(\right.$ Orchards,$\left.p_{1}\right)$ and thus we have (Cereals $\wedge$ Orchards, $\left.p_{1}\right)$.

Assume now that we also have the formula ( $\neg$ Orchards $\vee \neg$ Cereals, $\top$ ). This formula means that for any parcel, Orchards and Cereals are mutually exclusive. Such a piece of information has not to be understood as an encoding of a terminological knowledge coming from an ontology but as some practical knowledge stating that there is no parcel containing both cereals and orchards. Then one can now deduce that $\left(\perp, p_{1}\right)$ which expresses inconsistency about the information pertaining to $p_{1}$.

Note that the inconsistency in the above example does not affect the information $\left(\right.$ Orchards, $\left.p_{2}\right)$ pertaining to $p_{2}$. The reification allows us to keep inconsistency local.

Besides, we can observe in the above example that (Orchards, $\left.p_{1} \vee p_{2}\right)$ does not mean that there are orchards in $p_{1}$ or in $p_{2}$. This latter piece of information would be expressed by the disjunction (Orchards, $\left.p_{1}\right) \vee\left(\right.$ Orchards, $\left.p_{2}\right)$. Similarly, $\left(\neg\right.$ Orchards, $\left.p_{1} \vee p_{2}\right)$ means that neither in $p_{1}$ nor in $p_{2}$ there are orchards, while $\neg\left(\right.$ Orchards, $\left.p_{1} \vee p_{2}\right)$ means that at least one parcel of $\left\{p_{1}, p_{2}\right\}$ has no orchards.

The reified attributive formulas considered above are similar to the ones encountered in a recent multi-agent extension of possibilistic logic [15]. Here, a parcel plays the role of an agent. Namely, here instead of representing that 
any agent in a subset has some belief $\varphi$, we express that $\varphi$ is true for any elementary parcel in a subset. As in the multi-agent case, this formalism can be extended in order to handle uncertainty of formulas, as well as existential quantification on subsets of parcels.

\subsection{Uncertain attributive formulas}

Our attributive formula-based language is now extended in a possibilistic logic manner, by allowing uncertainty on properties. Let us recall that a standard propositional possibilistic formula [12] is a pair made of a logical proposition (which can be only true or false), associated with a certainty level. More precisely, the semantic counterpart of a possibilistic formula $(\varphi, \alpha)$ is a constraint $N(\varphi) \geq \alpha$ expressing that $\alpha$ is a lower bound on the necessity measure $N$ [13] of logical formula $\varphi$, which expresses available knowledge. Possibilistic logic has been proved to be sound and complete with respect to a semantics expressed in terms of the greatest possibility distribution $\pi$ underlying $N\left(N(\varphi)=1-\sup _{\omega \models \neg \varphi} \pi(\omega)\right)$. This distribution rank-orders interpretations according to their plausibility [12] (see section 6.1 for the general expression of such a distribution $\pi$ associated with a possibilistic logic base).

Note that a possibilistic formula $(\varphi, \alpha)$ can be viewed at the meta level as being only true or false, since either $N(\varphi) \geq \alpha$ or $N(\varphi)<\alpha$. Thus, this allows us to introduce possibilistic formula instead of propositional formula inside our attributive pair, and leads to the following definition.

Definition 4 (uncertain attributive formula) $A n$ uncertain attributive formula is a pair $((\varphi, \alpha), p)$ meaning that for the set of elementary parcels that satisfy $p$, the formula $\varphi$ is certain at least at level $\alpha$.

The inference rules of possibilistic logic [12] straightforwardly extend into the following rules for reasoning with uncertain attributive formulas:

\section{Proposition 3 (inference rules on uncertain attributive formulas)}

1. $\left(\left(\neg \varphi \vee \varphi^{\prime}, \alpha\right), p\right),\left(\left(\varphi \vee \varphi^{\prime \prime}, \beta\right), p^{\prime}\right) \vdash\left(\left(\varphi^{\prime} \vee \varphi^{\prime \prime}, \min (\alpha, \beta)\right), p \wedge p^{\prime}\right)$

2. $((\varphi, \alpha), p),\left(\left(\varphi^{\prime}, \beta\right), p\right) \vdash\left(\left(\varphi \wedge \varphi^{\prime}, \min (\alpha, \beta)\right), p\right)$

3.A. $((\varphi, \alpha), p),\left((\varphi, \beta), p^{\prime}\right) \vdash\left((\varphi, \min (\alpha, \beta)), p \vee p^{\prime}\right)$

3.B. $((\varphi, \alpha), p),\left((\varphi, \beta), p^{\prime}\right) \vdash\left((\varphi, \max (\alpha, \beta)), p \wedge p^{\prime}\right)$

4. if $p \vdash p^{\prime}$ then $\left((\varphi, \alpha), p^{\prime}\right) \vdash((\varphi, \alpha), p)$

5. if $\varphi \vdash \varphi^{\prime}$ then $((\varphi, \alpha), p) \vdash\left(\left(\varphi^{\prime}, \alpha\right), p\right)$

The fourth and the third rules (3.B. and 3.A.) correspond respectively to the fact that either i) we locate ourselves in the parcels that satisfy both $p$ and $p^{\prime}$ and then the certainty level of the formula $\varphi$ can reach the maximal upper 
bound of the certainty levels known in $p$ or in $p^{\prime}$, or that ii) we consider any parcel in the union of the models of $p$ and $p^{\prime}$ and then the certainty level is only guaranteed to be greater than the minimum of $\alpha$ and $\beta$.

Note that this representation formalism allows us, in particular, to express a greater uncertainty about a rather specific label for a parcel than about a more general label as in the following example.

Example 5 In order to express that parcel $p_{1}$ has either orchards or ornamental trees and more plausibly orchards, we can use the two uncertain attributive formulas with $p_{1}$ : ((Orchards, $\left.\left.\alpha_{1}\right), p_{1}\right)$ and ((Ornamental_trees $\vee$ Orchards, $\left.\left.\alpha_{2}\right), p_{1}\right)$ where $\alpha_{1} \leq \alpha_{2}$. At the semantic level, this is represented by the possibility distribution associated with $p_{1}$ :

$$
\pi(\omega)= \begin{cases}1 & \text { if } \omega \models \text { Orchards } \\ 1-\alpha_{1}<1 & \text { if } \omega \models \text { Ornamental_trees } \wedge \neg \text { Orchards } \\ 1-\alpha_{2} & \text { otherwise. }\end{cases}
$$

Suppose now, that parcel $p_{2}$ has almost certainly woods and more plausibly orchards, knowing that orchards are woods. This can be represented by the possibility distribution associated with $p_{2}$ :

$$
\pi(\omega)= \begin{cases}0 & \text { if } \omega \models \text { Orchards } \wedge \neg \text { Woods } \\ 1-\alpha_{2} & \text { if } \omega \models \neg \text { Orchards } \wedge \neg \text { Woods } \\ 1-\alpha_{1} & \text { if } \omega \models \neg \text { Orchards } \wedge \text { Woods }, \\ 1 & \text { if } \omega \models \text { Orchards } \wedge \text { Woods }\end{cases}
$$

This distribution can be syntactically encoded by the three formulas ( $\neg$ Orchards $\vee$ Woods, 1$), \top),\left(\left(\right.\right.$ Woods,$\left.\left.\alpha_{2}\right), p_{2}\right)$ and ((Orchards, $\left.\left.\alpha_{1}\right), p_{2}\right)$, with $\alpha_{2} \geq \alpha_{1}$.

\subsection{Existential quantification of parcels}

In the previous part of the section, we have considered attributive pairs $(\varphi, p)$ as formulas expressing that in each parcel satisfying $p, \varphi$ holds. Now, we may want to express that $\varphi$ holds at least in one parcel satisfying $p$.

Definition 5 (existential attributive formula) We denote by a pair $(\varphi,[p])$ the formula expressing that $\varphi$ holds at least in one parcel satisfying $p$. This formula is logically equivalent to $\neg(\neg \varphi, p)$. 
We can check that the following inference rules hold:

\section{Proposition 4}

1. $(\neg \varphi \vee \psi, p),(\varphi,[p]) \vdash(\psi,[p])$

2. $(\neg \varphi \vee \psi, p),(\varphi \vee \rho,[p]) \vdash(\psi \vee \rho,[p])$

Proof : 1. Let us assume that $(\neg \varphi \vee \psi, p)$ and $(\varphi,[p])$ and that $(\neg \psi, p)$. Then using resolution rule of Property 2.1 with the first and third formulas, we get $(\neg \varphi, p)$. Now, $(\varphi,[p])$ can be translated into $\neg(\neg \varphi, p)$. Hence, we obtain a contradiction. This means that the hypothesis that $(\neg \psi, p)$ holds when $(\neg \varphi \vee \psi, p)$ and $(\varphi,[p])$ holds is absurd. Thus, $(\neg \varphi \vee \psi, p)$ and $(\varphi,[p])$ implies $(\neg(\neg \psi, p)$, i.e., $(\psi,[p])$.

2. Let us suppose that $(\neg \varphi \vee \psi, p)$ and $(\varphi \vee \rho,[p])$. By definition, $(\varphi \vee \rho,[p])$ is equivalent to $\neg(\neg \varphi \wedge \neg \rho, p)$. Using the contrapositive of Property 2.2, we get $\neg(\neg \varphi, p) \vee \neg(\neg \rho, p)$, that is equivalent by definition to $(\varphi,[p]) \vee(\rho,[p])$. Using Property 4.1 between $(\neg \varphi \vee \psi, p)$ and $(\varphi,[p]) \vee(\rho,[p])$, we get $(\psi,[p]) \vee$ $(\rho,[p])$. This is equivalent by definition to $\neg(\neg \psi, p) \vee \neg(\neg \rho, p)$. Knowing that $\neg \psi \wedge \neg \rho \vdash \neg \psi$, using Property 2.5, we get that $(\neg \psi \wedge \neg \rho, p) \vdash(\neg \psi, p)$, similarly we can obtain that $(\neg \psi \wedge \neg \rho, p) \vdash(\neg \rho, p)$. Hence that $(\neg \psi \wedge \neg \rho, p) \vdash$ $(\neg \psi, p) \wedge(\neg \rho, p)$. By contraposition, we get $\neg(\neg \psi, p) \vee \neg(\neg \rho, p) \vdash \neg(\neg \psi \wedge$ $\neg \rho, p)$, that is equivalent by definition to $(\psi \vee \rho,[p])$.

Note that taking $\varphi=\top$ in Proposition 4.1 we obtain that:

$$
\text { If } \neg(\perp, p) \text { then }(\psi, p) \vdash(\psi,[p])
$$

which means that if the information about $p$ is not inconsistent, one can weaken it existentially on parcels.

The above inference rules clearly extend to the case where information is uncertain. Indeed we have:

$$
((\neg \varphi \vee \psi, \alpha), p),((\varphi \vee \rho, \beta),[p]) \vdash((\psi \vee \rho, \min (\alpha, \beta)),[p])
$$

Moreover, keeping in mind that $((\varphi, \alpha), p)$ means that at least in the elementary parcels satisfying $p$ (it may be the case for other parcels also), $\varphi$ is certain at least at level $\alpha$, one can as well express that at most in the elementary parcels satisfying $p, \varphi$ is certain at least at level $\alpha$, by the expression $\neg((\varphi, \alpha),[\neg p])$. This last expression indeed means that it is not the case that $\varphi$ is certain at least at level $\alpha$ in the elementary parcels outside $p$ (the models of $\neg p$ are the elementary parcels that does not satisfy $p$ ). Note that the expression $\neg((\varphi, \alpha),[\neg p])$ is thus equivalent to $(\neg(\varphi, \alpha), \neg p)$, which precisely states that $\varphi$ is not at least $\alpha$-certain for any elementary parcel outside $p$. 


\subsection{Localization of attributive knowledge}

We have seen that geographical information systems should handle two kinds of information : ontological information pertaining to property hierarchies or to spatial organization on the one hand, and attributive information linking parcels to properties on the other hand. Still, attributive information itself may have two different intended meaning, namely when stating $(\varphi, p)$ one may want to express:

- that everywhere in each parcel satisfying $p, \varphi$ holds as true. For instance, in such a case, (Vegetation, $p$ ) cannot be consistent with (Lakes, $p$ ) since "Vegetation" and "Lakes" are mutually exclusive in the ontology of Figure 1 .

- that somewhere in each parcel satisfying $p, \varphi$ holds as true. In that case, the two previous formulas should not be considered as inconsistent, in spite of the ontology, since in each parcel of $p$ there may exist different areas covered by "Vegetation" and "Lakes" respectively.

Note that the two above situations should not be confused with still another case where two distinct mutually exclusive labels such as "Vegetation" and "Lakes" might be attached to the same area because they are intimately mixed in this area, as in a "swamp". This latter case should be handled by adding a new appropriate label in the ontology.

More formally, viewing an elementary parcel $p$ as a collection of more elementary objects $o$, when we assert $(\varphi, p$, somewhere $)$, it really means that $\exists o \in$ $p, \varphi(o)$. If the parcel $p$ is not elementary then the formula $(\varphi, p$, somewhere $)$ has to be understood as for all $p^{\prime}$ such that $p^{\prime} \vdash p,\left(\varphi, p^{\prime}\right.$, somewhere $)$ holds. Thus, it should be clear that all the inference rules of Proposition 2, which hold for the "everywhere" understanding, no longer necessarily hold in the "somewhere" reading. Indeed, the inference rule $2.2(\varphi, p),(\psi, p) \vdash(\varphi \wedge \psi, p)$ is no longer compatible with this reading of attributive formulas since $\exists o \in p, \varphi(o)$ and $\exists o^{\prime} \in p, \psi\left(o^{\prime}\right)$ does not entail $\exists o^{\prime \prime} \in p, \varphi\left(o^{\prime \prime}\right) \wedge \psi\left(o^{\prime \prime}\right)$. More generally, here are the counterparts of proposition 2 that holds for the "somewhere" reading:

\section{Proposition 5 (inference rules on attributive formulas)}

1'. $\left(\neg \varphi \vee \varphi^{\prime}, p \wedge p^{\prime}, e\right),\left(\varphi \vee \varphi^{\prime \prime}, p^{\prime}, s\right) \vdash\left(\varphi^{\prime} \vee \varphi^{\prime \prime}, p \wedge p^{\prime}, s\right)$

2'. $(\varphi, p, s),\left(\varphi^{\prime}, p, e\right) \vdash\left(\varphi \wedge \varphi^{\prime}, p, s\right)$

3'. $(\varphi, p, s),\left(\varphi, p^{\prime}, s\right) \vdash\left(\varphi, p \vee p^{\prime}, s\right)$

4'. if $p^{\prime} \vdash p$ then $(\varphi, p, s) \vdash\left(\varphi, p^{\prime}, s\right)$

5'. if $\varphi \vdash \varphi^{\prime}$ then $(\varphi, p, s) \vdash\left(\varphi^{\prime}, p, s\right)$ where $(\varphi, p, s)$ stands for all $p^{\prime}$ such that $p^{\prime} \vdash p \exists o \in p^{\prime}, \varphi(o)$, and $(\varphi, p, e)$ for $\forall o \in p, \varphi(o)$.

Moreover, we have the following relation between "somewhere" and "everywhere" formulas: 
$6^{\prime} . \neg(\varphi, p, s) \equiv(\neg \varphi, p, e)$

Moreover, the terminological knowledge encoded by property or spatial ontologies also interferes with the "somewhere" and "everywhere" readings of attributive formulas. Terminological information and attributive information should be handled separately, not only because they refer to two different types of information but, more importantly, because terminological distinctions expressed by mutual exclusiveness of labels do not mean that these distinct labels cannot be simultaneously true in a given area. For instance, saying that "Orchards" are not "Meadows" and conversely, does not prevent to have both "Meadows" and "Orchards" on the same parcel. But it would become forbidden if we had the strongly restrictive assumption that the size of the parcels are adapted to the leaves of the property ontology in such a way that any elementary parcel cannot be associated to several mutually exclusive properties (of the same ontology). Under this latter assumption, the "somewhere" and "everywhere" readings would be equivalent. In other words, mutual exclusiveness between ontology labels (e.g $a \leftrightarrow \neg b$ where $a$ and $b$ are propositional symbols of $\mathscr{V}_{i}$ coming from the same ontology of properties) has not the same meaning as its counterpart put in an attributive form $(a \leftrightarrow \neg b, \top)$ applied universally (i.e., with the "everywhere" reading) to each parcel, since it may happen that for a parcel $p$, we have $(a, p) \wedge(b, p)$ (with a "somewhere" reading).

This is why it is not possible to mix mutual exclusiveness of terminological labels of an ontology with attributive knowledge in general. Indeed, ontological mutual exclusiveness of $\varphi$ and $\psi$ means that $\forall o, \varphi(o) \leftrightarrow \neg \psi(o)$. But it does not prevent from having $(\varphi, p) \wedge(\psi, p)$, since the latter means $\exists o \in p, \varphi(o) \wedge \exists o^{\prime} \in$ $p, \psi\left(o^{\prime}\right)$, and, in general, $p$ may contain at least two distinct objects $o$ and $o^{\prime}$.

However, subsumption properties can be added to attributive formulas without any problem. Indeed $\varphi \vdash \psi$ means $\forall o, \varphi(o) \rightarrow \psi(o)$, and if we have $(\varphi, p)$, implicitly meaning that $\exists o \in p, \varphi(o)$, then we obtain $\exists o \in p, \psi(o)$, i.e., $(\psi, p)$. Thus we can write the subsumption property as $(\varphi \rightarrow \psi, \top)$.

\section{Information fusion}

Spatial information may be provided by different sources. We assume in the following that these sources are equally reliable. However these sources may or may not use the same ontology of properties and the same spatial ontology. Generally speaking, fusing consistent knowledge bases does not raise any special problem since it amounts to infer from the union of the knowledge bases, i.e, apply inference rules such as the ones of Proposition 2 (or Proposition 3 in case of uncertainty) and Proposition 5 (for the "somewhere" formulas). In presence of inconsistency, another combination process should be defined and 
used.

In this section, we first provide a short background on information fusion and its possibilistic logic encoding, before discussing the problems raised by the use of different ontologies by the sources. Finally, we develop an example where two sources use the same property ontology for spatial information but different space partitions and express information in the logical representational language introduced in section 4 .

\subsection{Background on fusion in possibilistic logic}

Suppose we have two pieces of information $\varphi$ and $\psi$ provided by two different sources. If $\varphi$ and $\psi$ are consistent, the fusion is straightforward and yields the conjunction $\varphi \wedge \psi$. If $\varphi \wedge \psi \equiv \perp$ then at least one of the two sources should be wrong. But, if we do not want to throw away all pieces of information, one may still assume that one is right, this yielding the disjunction $\varphi \vee \psi$ as a result of the fusion. In case of inconsistency, instead of building a disjunction, another possibility would be to weaken the "everywhere" reading leading to inconsistency into a "somewhere" reading. In the particular case of an inconsistency due to a mutual exclusiveness constraint coming from an ontology, still another way of getting rid of this inconsistency is to introduce new labels in the ontology which would be compatible with the apparently conflicting labels (as in the "swamp" example above). This latter option has been proposed by Doukari and Jeansoulin [11].

In standard possibilistic logic, conflict becomes a matter of degree. Let us assume that the information provided by the two sources can be represented by two possibilistic logic bases $\Sigma_{1}$ and $\Sigma_{2}$ where $\Sigma_{1}=\left\{\left(\varphi_{i}, \alpha_{i}\right), i=1, \ldots, m\right\}$ and $\Sigma_{2}=\left\{\left(\psi_{j}, \beta_{j}\right), i=j, \ldots, n\right\} . \Sigma_{1}$ can be semantically associated with a possibility distribution [12] $\pi_{1}$ such that:

$$
\pi_{1}(\omega)= \begin{cases}1 & \text { if } \omega \models \varphi_{1} \wedge \cdots \wedge \varphi_{m}, \\ 1-\max \left\{\alpha_{i} \text { s.t. }\left(\varphi_{i}, \alpha_{i}\right) \in \Sigma_{1}, \omega \models \neg \varphi_{i}\right\} & \text { otherwise }\end{cases}
$$

The possibility distribution $\pi_{2}$ associated with $\Sigma_{2}$ is similarly defined. Thus, the conjunctive combination $\Sigma_{1} \cup \Sigma_{2}$ will be associated with $\pi_{\Sigma_{1} \cup \Sigma_{2}}=\min \left(\pi_{1}, \pi_{2}\right)$. When $\nexists \omega$, s.t. $\pi_{\Sigma_{1} \cup \Sigma_{2}}(\omega)=1$, this means that $\varphi_{1} \wedge \cdots \wedge \varphi_{m} \wedge \psi_{1} \wedge \cdots \wedge \psi_{n} \equiv \perp$. Let $h g t(\pi)=\max _{\omega} \pi(\omega)$ be the height of a possibility distribution $\pi$. Then, the level of inconsistency of $\Sigma_{1} \cup \Sigma_{2}$, denoted $\operatorname{Inc}\left(\Sigma_{1} \cup \Sigma_{2}\right)$, is defined by $\operatorname{Inc}\left(\Sigma_{1} \cup \Sigma_{2}\right)=1-\operatorname{hgt}\left(\min \left(\pi_{1}, \pi_{2}\right)\right)$. The subset of all the formulas in $\Sigma_{1} \cup \Sigma_{2}$ with a certainty level strictly greater than $\operatorname{Inc}\left(\Sigma_{1} \cup \Sigma_{2}\right)$ is consistent [12]. 
This leads to a renormalized conjunction mode defined by

$\pi_{\text {renorm.conj. }}(\omega)= \begin{cases}\min \left(\pi_{1}(\omega), \pi_{2}(\omega)\right) & \text { if } \min \left(\pi_{1}(\omega), \pi_{2}(\omega)\right)<\operatorname{hgt}\left(\min \left(\pi_{1}, \pi_{2}\right)\right) \\ 1 & \text { otherwise }\end{cases}$

Thus, a natural idea underlying so-called adaptive fusion operators for two possibility distributions $\pi_{1}$ and $\pi_{2}$, is to look for combination operations parameterized with the level of inconsistency Inc. These operators are such that if $I n c=0$ the conjunctive min-based combination of the distributions is retrieved, and if $I n c=1$ the disjunctive max-based combination of the distributions is obtained. Two examples of such a rule are [16]:

$$
\begin{aligned}
& \pi_{\text {adapt }}(\omega)=\max \left(\frac{\min \left(\pi_{1}(\omega), \pi_{2}(\omega)\right)}{h g t\left(\min \left(\pi_{1}, \pi_{2}\right)\right)}, \min \left(\max \left(\pi_{1}(\omega), \pi_{2}(\omega)\right), \operatorname{hgt}\left(\min \left(\pi_{1}, \pi_{2}\right)\right)\right)\right) \\
& \pi_{\text {med }}(\omega)=\min \left(\max \left(\pi_{1}, \pi_{2}\right), \max \left(\pi_{1}, \text { Inc }\right), \max \left(\pi_{2}, \text { Inc }\right)\right)
\end{aligned}
$$

The first rule provides a distribution that is always normalized (thus the rule gets rid of inconsistency). It is not the case of the second one which is just a median operation.

Besides, the syntactic counterpart of the pointwise combination of two possibility distributions $\pi_{1}$ and $\pi_{2}$ into a distribution $\pi_{1} \oplus \pi_{2}$ by any monotonic ${ }^{1}$ combination operator $\oplus$ such that $1 \oplus 1=1$, can be easily computed. Namely, if $\Sigma_{1}$ is associated with $\pi_{1}$ and $\Sigma_{2}$ with $\pi_{2}$, a possibilistic base that is semantically equivalent to $\pi_{1} \oplus \pi_{2}$ can be computed as [6]:

$$
\Sigma_{1 \oplus 2}=\mid \begin{array}{cl}
\left\{\left(\varphi_{i}, 1-\left(1-\alpha_{i}\right) \oplus 1\right)\right. & \text { s.t. } \left.\left(\varphi_{i}, \alpha_{i}\right) \in \Sigma_{1}\right\}, \\
\cup\left\{\left(\psi_{j}, 1-1 \oplus\left(1-\beta_{j}\right)\right)\right. & \text { s.t. } \left.\left(\psi_{j}, \beta_{j}\right) \in \Sigma_{2}\right\}, \\
\cup\left\{\left(\varphi_{i} \vee \psi_{j}, 1-\left(1-\alpha_{i}\right) \oplus\left(1-\beta_{j}\right)\right)\right. & \text { s.t. } \left.\left(\varphi_{i}, \alpha_{i}\right) \in \Sigma_{1},\left(\psi_{j}, \beta_{j}\right) \in \Sigma_{2}\right\} .
\end{array}
$$

For $\oplus=\min$, we get $\pi_{\Sigma_{1} \cup \Sigma_{2}}=\min \left(\pi_{1}, \pi_{2}\right)$ as expected. For $\oplus=\max$, we get $\Sigma_{\max \left(\pi_{1}, \pi_{2}\right)}=\left\{\left(\varphi_{i} \vee \psi_{j}, \min \left(\alpha_{i}, \beta_{j}\right)\right)\right.$ s.t. $\left(\varphi_{i}, \alpha_{i}\right) \in \Sigma_{1}$, and $\left.\left(\psi_{j}, \beta_{j}\right) \in \Sigma_{2}\right\}$.

\subsection{Spatial information fusion - A general discussion}

Possibilistic information fusion, as just recalled, easily extends to attributive formulas expressing spatial information. Indeed, each formula $(\varphi, p)$ provided by a source is equivalent to the conjunction of formulas $\left(\varphi, p_{i}\right)$, where the $p_{i}$ 's correspond to the leaves of the spatial ontology used by this source and $p_{i} \models p$.

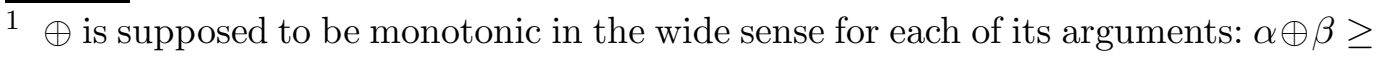
$\gamma \oplus \delta$ as soon as $\alpha \geq \gamma$ and $\beta \geq \delta$.
} 
In section 3, we have advocated the idea that different spatial ontologies should at least share the same leaves (called elementary parcels). This last assumption is justified by the fact that it is always possible, starting from the two spatial partitions $\mathscr{P}_{1}$ and $\mathscr{P}_{2}$ made by the leaves of two distinct spatial ontologies $G_{s 1}$ and $G_{s 2}$, to build two new refined ontologies whose leaves are the elements of the refined partition induced by the two initial partitions $\mathscr{P}_{1}$ and $\mathscr{P}_{2}$ (by taking the non-empty intersection of pairs of elementary parcels of the two partitions). See Figure 3 for an example of refinement:

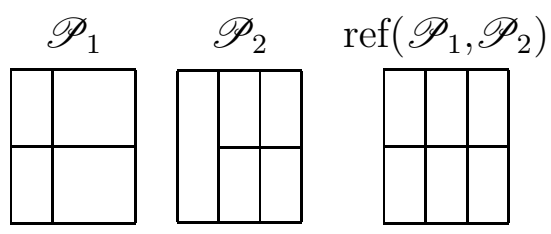

Fig. 3. Example of refinement of $\mathscr{P}_{1}$ and $\mathscr{P}_{2}$

Then, for each elementary parcel $p_{i}$ possibilistic information fusion takes place. However, if the two sources do not use the same property ontology, an additional source of knowledge laying bare the links between the two vocabularies is necessary in order to determine if for a given elementary parcel the sources are conflicting or not. This is specially important if the fusion principle depends on the existence and the extent of inconsistency between the sources.

Moreover, in case of several property ontologies, it may be the case that even with the additional source of knowledge linking them, it is not always possible to find a logical expression expressed in a vocabulary that is equivalent to another expression in the other vocabulary. In the general case, only approximations can be found, if the result of the fusion should be communicated to a user in one vocabulary rather than in the other.

Although the ontology-based examples chosen may have suggested that our approach is dedicated to the processing of symbolic information, it also applies to the particular case of numerical information. Assume that $(\theta \leq x \leq \rho, p)$ denotes the piece of information that for any elementary parcel satisfying $p$, the parameter $x$ (e.g., the height of rain for some time period) is in between values $\theta$ and $\rho$. Then the following entailments directly follow from the inference rules given in Proposition 2:

$$
(\theta \leq x \leq \rho, p),(\sigma \leq x \leq \tau, p) \vdash(\max (\theta, \sigma) \leq x \leq \min (\rho, \tau), p)
$$

and more generally (provided that the bounds are consistent)

$$
\begin{aligned}
& (\theta \leq x \leq \rho, p),\left(\sigma \leq x \leq \tau, p^{\prime}\right) \vdash\left(\max (\theta, \sigma) \leq x \leq \min (\rho, \tau), p \wedge p^{\prime}\right) \\
& (\theta \leq x \leq \rho, p),\left(\sigma \leq x \leq \tau, p^{\prime}\right) \vdash\left(\min (\theta, \sigma) \leq x \leq \max (\rho, \tau), p \vee p^{\prime}\right)
\end{aligned}
$$

It is worth noticing that the two above rules are at the basis of C-calculus $[9,8]$ 


\begin{tabular}{|l|l|}
\hline$p_{1}$ & $p_{2}$ \\
\hline$p_{3}$ & $p_{4}$ \\
\hline
\end{tabular}

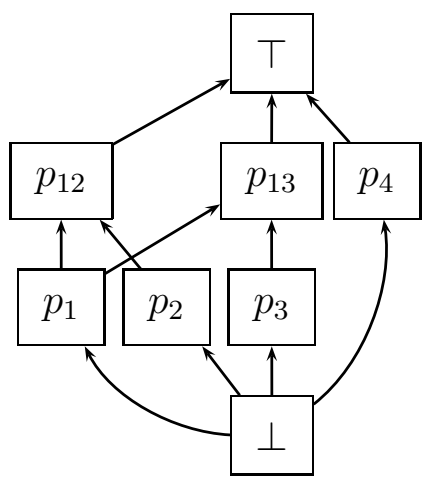

Fig. 4. Partitioned area and spatial ontology used in the example

for propagating interval-valued parameter estimates associated with elements of partitions, when refining or coarsening the partitions.

\subsection{Illustrative Example}

We here consider an example inspired from [28], where two sources report observations about an area which is partitioned in four elementary parcels organized into a spatial ontology as pictured in Figure 4. We suppose that both sources are using the property ontology given in Figure 1. The spatial ontology given in Figure 4 and the property ontology lead to have the following formulas that holds:

Spatial formulas
(1) $p_{1} \rightarrow p_{12}$
(2) $p_{1} \rightarrow p_{13}$,
(3) $p_{2} \rightarrow p_{12}$,
(4) $p_{3} \rightarrow p_{13}$,
(5) $p_{12} \vee p_{13} \vee p_{4}$,
(6) $p_{12} \rightarrow p_{1} \vee p_{2}$
(7) $p_{13} \rightarrow p_{1} \vee p_{3}$
(8) $p_{1} \wedge p_{2} \rightarrow \perp$
(9) $p_{1} \wedge p_{3} \rightarrow \perp$
(10) $p_{1} \wedge p_{4} \rightarrow \perp$
(11) $p_{2} \wedge p_{3} \rightarrow \perp$
(12) $p_{2} \wedge p_{4} \rightarrow \perp$
(13) $p_{3} \wedge p_{4} \rightarrow \perp$

Property formulas

(14) Meadows $\rightarrow$ Vegetation,

(15) Agr. areas $\rightarrow$ Vegetation,

(16) Woods $\rightarrow$ Vegetation,

(17) Orchards $\rightarrow$ Woods,

(18) Orchards $\rightarrow$ Agr. areas,

(19) Water $\rightarrow$ Land cover,

(20) Vegetation $\rightarrow$ Land Cover,

(21) Rivers $\rightarrow$ Water,

(22) Cereals $\rightarrow$ Agr. areas

(23) Cereals $\wedge$ Orchards $\rightarrow \perp$,

(24) Cereals $\wedge$ Meadows $\rightarrow \perp$,

(25) Woods $\wedge$ Meadows $\rightarrow \perp$

The information provided by the two sources is supposed to be the one given in Figure 5.

Presented as such, the example is ambiguous, because when there are two labels on a parcel, we do not know if they are connected by a conjunction 
Source 1

\begin{tabular}{|l|l|}
\hline $\begin{array}{l}\text { Agr. areas } \\
\text { Meadows }\end{array}$ & $\begin{array}{l}\text { Orchards } \\
\text { Woods }\end{array}$ \\
\cline { 2 - 2 } & Water \\
\hline
\end{tabular}

Source 2

\begin{tabular}{|c|l|}
\hline \multicolumn{2}{|c|}{ Woods } \\
\hline $\begin{array}{c}\text { Cereals } \\
\text { Meadows }\end{array}$ & Rivers \\
\hline
\end{tabular}

Fig. 5. The information given by the sources

(meaning that several labels apply simultaneously to the parcel,) or by a disjunction (meaning that one does not know what is the right label, but one of them applies to the parcel). Similarly, when a label applies to a union of elementary parcels, one may wonder if the label apply to each elementary parcel or, maybe to some of them without knowing which of them. Clearly, this leads to four logical readings of two labels $a$ and $b$ associated with an area covered by two elementary parcels $p_{1}$ and $p_{2}$ :

(i.) $\left(a \wedge b, p_{1} \vee p_{2}\right)$. This means that both $a$ and $b$ apply to each of $p_{1}$ and $p_{2}$.

(ii.) $\left(a \wedge b, p_{1}\right) \vee\left(a \wedge b, p_{2}\right)$. This means that both $a$ and $b$ apply to $p_{1}$ or both $a$ and $b$ apply to $p_{2}$.

(iii.) $\left(a \vee b, p_{1} \vee p_{2}\right)$. This means that $a$ applies to each of $p_{1}$ and $p_{2}$ or $b$ applies to each of $p_{1}$ and $p_{2}$.

(iv.) $\left(a \vee b, p_{1}\right) \vee\left(a \vee b, p_{2}\right)$. This is the most imprecise case where we do not know what of $a$ or $b$ applies to what of $p_{1}$ or $p_{2}$. This last case may be particularized by adding the mutual exclusiveness constraint $\neg\left(a, p_{1} \vee\right.$ $\left.p_{2}\right) \wedge \neg\left(b, p_{1} \vee p_{2}\right)$ specifying that each label cannot apply to both parcels.

Note that there is another latent ambiguity, when a label is attached to a parcel, regarding the localization of how the label applies to the parcel. As discussed in section 4.4, the label may apply everywhere or only somewhere in the parcel. Observe that in the first above reading if $a$ and $b$ are mutually exclusive (e.g. "Meadows" and "Agr. areas") the everywhere understanding is impossible (if we admit that sources provide consistent information).

A third kind of ambiguity when understanding the example, is about to know if the "closed world assumption" (CWA) holds or not: does a source provide the complete list of possible properties of a considered parcel? For instance, if a source says that a parcel contains "Meadows" and "Cereals" does it exclude that the parcel would also include "Woods"? "Woods" would be indeed excluded by applying CWA. Note that the application of the "closed world assumption" may help to induce "everywhere" information from "somewhere" information. Indeed, if we know that all formulas attached to $p$ are $\varphi_{1}, \ldots$, and $\varphi_{n}$ with a somewhere meaning (i.e., $\left.\left(\varphi_{1}, p, \mathrm{~s}\right) \wedge \ldots \wedge\left(\varphi_{n}, p, \mathrm{~s}\right)\right)$, the "closed world assumption" allows us to jump to the conclusion that $\left(\bigvee_{i=1, n} \varphi_{i}, p\right.$, e). Since, if the only thing we know about $p$ is that $\varphi_{1}, \ldots, \varphi_{n}$ hold somewhere, 
the close world assumption entails that if there were another property that holds somewhere else in $p$, it would have been already said, hence in $p$ the only properties that hold are $\varphi_{1}, \ldots, \varphi_{n}$.

In the following, we are going to only consider non ambiguous understandings of the example, still illustrating the main points above, under the "closed world assumption". Namely we use the following reading of the example:

\section{Source 1}

Source 2

(26) (Agr. areas, $\left.p_{13}, \mathrm{~s}\right)$

(27) (Meadows, $p_{13}, \mathrm{~s}$ )

(28) (Orchards, $p_{2}, \mathrm{~s}$ )

(29) (Woods, $p_{2}, \mathrm{~s}$ )

(30) (Water, $p_{4}, \mathrm{~s}$ )

(31) (Woods, $p_{12}, \mathrm{~s}$ )

(32) (Cereals, $\left.p_{3}, \mathrm{~s}\right)$

(33) (Meadows, $p_{3}, \mathrm{~s}$ )

(34) (Rivers, $p_{4}, \mathrm{~s}$ )

In order to be able to merge pieces of information associated to $p_{13}$, and $p_{12}$, we should project the information on more elementary parcels (here $p_{1} \equiv$ $p_{12} \wedge p_{13}$ ). Using formula $7: p_{13} \rightarrow p_{1} \vee p_{3}$ and the inference rule 4' (with $p_{1} \vdash p_{13}$ and $p_{3} \vdash p_{13}$ ), and similarly for $p_{12}$, we obtain the following formulas:

Source 1

Source 2

(35) (Agr. areas, $\left.p_{1}, \mathrm{~s}\right)$

(36) (Agr. areas, $\left.p_{3}, \mathrm{~s}\right)$

(39) (Woods, $p_{1}, \mathrm{~s}$ )

(37) (Meadows, $p_{1}, \mathrm{~s}$ )

(40) (Woods, $p_{2}, \mathrm{~s}$ )

(38) (Meadows, $p_{3}, \mathrm{~s}$ )

The "closed world assumption" allows us to deduce:

Source 1

(41) (Agr. areas $\vee$ Meadows, $p_{1}$, e)

(42) (Agr. areas $\vee$ Meadows, $p_{3}, \mathrm{e}$ )

(43) (Orchards $\vee$ Woods, $p_{2}$, e)

(44) (Water, $\left.p_{4}, \mathrm{e}\right)$
Source 2

(45) (Woods, $p_{1}$, e)

(46) (Woods, $\left.p_{2}, \mathrm{e}\right)$

(47) (Cereals $\vee$ Meadows, $p_{3}$, e)

(48) (Rivers, $\left.p_{4}, \mathrm{e}\right)$

Once the above deduction step is performed, one can proceed with the fusion step. Let us first consider the conjunctive mode. We obtain:

parcel $\mathbf{p}_{1}$ : For source 1 , we have formulas (35), (37), (41), and for source 2, (45). The conjunction of (41) and (45) yields (Orchards, $\left.p_{1}, e\right)$, which in turn leads to a contradiction with (37).

parcel $\mathbf{p}_{2}$ : For source 1, we have formulas (28), (29), (43), and for source 2, (40). The conjunction of these four formulas is consistent, and yields (Woods, $p_{2}$, e) $\wedge\left(\right.$ Orchards, $\left.p_{2}, \mathrm{~s}\right)$.

parcel $\mathbf{p}_{3}$ : For source 1, we have formulas (36), (38), (42), and for source 2, (32), (33),(47). The conjunction of these six formulas is consistent, and yields 
$\left(\right.$ Cereals $\vee$ Meadows, $p_{3}$, e $) \wedge\left(\right.$ Cereals, $\left.p_{3}, \mathrm{~s}\right) \wedge\left(\right.$ Meadows, $\left.p_{3}, \mathrm{~s}\right)$.

parcel $\mathbf{p}_{4}$ : For source 1, we have formula (44), and for source 2, (48). The conjunction of these two formulas is consistent, and yields (Rivers, $p_{4}, \mathrm{e}$ ).

These conclusions are summarized in the following figure.

\begin{tabular}{|l|l|}
\hline$\perp$ & $\begin{array}{l}(\text { Orchards }, s) \\
(\text { Woods }, e)\end{array}$ \\
\hline $\begin{array}{l}(\text { Cereals }, s) \\
(\text { Meadows }, s)\end{array}$ & $($ Rivers,$e)$ \\
\hline
\end{tabular}

Source 1 and source 2 are conflicting on $\mathrm{p}_{1}$, hence following Section 5.1, we are led to perform a disjunction of the two pieces of information, namely $((35) \wedge(37) \wedge(41)) \vee(45)$. It yields (Vegetation, $\left.\mathrm{p}_{1}, \mathrm{e}\right) \wedge($ Agr. areas $\vee$ Woods, $\left.\mathrm{p}_{1}, \mathrm{~s}\right) \wedge\left(\right.$ Meadows $\vee$ Woods, $\mathrm{p}_{1}, \mathrm{~s}$ ), taking advantage of the ontology (namely Vegetation $\equiv($ Agr. areas $\vee$ Woods $\vee$ Meadows $)$ ).

Observe that the conflict between two sources on a parcel may come from the fact that CWA has been applied to the information of each source prior the fusion. Thus, one may think of backtracking on the application of CWA when there is a conflict. Here, on $\mathrm{p}_{1}$, the induction from (Woods, $\mathrm{p}_{1}, \mathrm{~s}$ ) stated by source 2 , to (Woods, $\mathrm{p}_{1}$, e) is perhaps too adventurous. It can be checked that (Woods, $\left.\mathrm{p}_{1}, \mathrm{~s}\right) \wedge(35) \wedge(37) \wedge(41)$ is consistent and gives (Orchards $\vee$ Meadows, $\left.\mathrm{p}_{1}, \mathrm{e}\right) \wedge\left(\right.$ Orchards, $\left.\mathrm{p}_{1}, \mathrm{~s}\right) \wedge$ (Meadows, $\left.\mathrm{p}_{1}, \mathrm{~s}\right)$.

In their treatment of the kind of fusion problem exemplified above, Pham [28] and Jeansoulin et al. [23] distinguish between pessimistic and optimistic fusion modes. The "optimistic" fusion is characterized by the facts that the fusion of the pieces of information associated to the same parcel by two sources is obtained by conjunction. Clearly, this may lead to inconsistency on some parcels. In such a case the "pessimistic" mode would be the only one to provide consistent results (by assuming disjunction in place of conjunction in the fusion step. As can be seen, the framework proposed here basically agrees with the principles underlying the above-cited approach. However, their proposal relies on a representation manipulating sets of ontology labels (associated to parcels), using a specific information containment relation between sets of labels that extends the inheritance relation of the ontology. Our approach uses i) a pure logical representation setting (which allows to maintain an explicit distinction between conjunction and disjunction of labels), ii) distinguishes between "somewhere" and "everywhere" statements, iii) allows to express CWA (an assumption apparently made often tacitly), iv) applies the general setting of logic-based information fusion. The result of the fusion may be more precise with our approach, thanks to a greater expressivity power of the representa- 
tion framework (e.g., instead of just finding Vegetation on $\mathrm{p}_{1}$, while we found $\left(\right.$ Vegetation, $\left.\mathrm{p}_{1}, \mathrm{e}\right) \wedge\left(\right.$ Agr. areas $\vee$ Woods, $\left.\mathrm{p}_{1}, \mathrm{~s}\right) \wedge$ (Meadows $\vee$ Woods, $\mathrm{p}_{1}$, s)).

Our logical framework also allows us to have a possibilistic handling of uncertainty, and then a variety of combination operations, which may depend on the level of conflict between the sources, or on their relative priority [6], can be encoded, as recalled in Section 5.1. The uncertainty setting enables us to enrich the reading of the example. Consider the information given by source 1 on $\mathrm{p}_{2}$, namely "Orchards, Woods". As discussed in section 4.2, such an information may express that $\mathrm{p}_{2}$ is covered by Woods, and plausibly by Orchards. This can be understood in an "everywhere", or "somewhere" manner. With the "everywhere" reading, this can be syntactically encoded by the possibilistic formulas $\left((\right.$ Woods, 1$\left.), p_{2}, e\right)$ and $\left((\right.$ Orchards,$\left.\alpha), p_{2}, e\right)$, with $\alpha<1$, together with the ontology information $((\neg$ Orchards $\vee W o o d s, 1), \top)$. Similarly, the information given by source 2 on $\mathrm{p}_{2}$ can be encoded as $\left((\operatorname{Woods}, 1), p_{2}, e\right)$. Here, there is no inconsistency, hence ((Woods, 1$\left.), \mathrm{p}_{2}, \mathrm{e}\right) \wedge\left((\right.$ Orchards, $\left.\alpha), \mathrm{p}_{2}, \mathrm{e}\right)$.

Imagine that, now, source 2 says $\left((W o o d s, 1), p_{2}, e\right)$ and $\left((\right.$ Orn.trees,$\left.\beta), p_{2}, e\right)$. The two sources are now partially inconsistent on $\mathrm{p}_{2}$, and it can be checked that the level of inconsistency of the information provided by the two sources, about $\mathrm{p}_{2}$, is $\operatorname{Inc}=\min (\alpha, \beta)$.

Different fusion modes can be used, corresponding to different attitudes with respect to a conflict. For instance, one can use a renormalized conjunction (based on min see section 5.1) that gets rid of the conflict. Namely, the syntactic counterpart of this operator yields, if we assume $\alpha>\beta,\left((\right.$ Woods, 1$\left.), p_{2}, e\right) \wedge$ $\left((\right.$ Orchards,$\left.\alpha), p_{2}, e\right)$.

If we choose a disjunctive attitude (based on $\oplus=\max$ see end of section 5.1), one gets $\left((W o o d s, 1), p_{2}, e\right) \wedge\left((\right.$ Orchards $\vee$ Orn. trees,$\left.\beta), p_{2}, e\right)$.

In case we again combine the two previous results obtained with the above fusion modes, by a product-based conjunction $(\oplus=$ product, see end of section $5.1)$, one would obtain $\left((\right.$ Woods, 1$\left.), p_{2}, e\right) \wedge(($ Orchards $\vee$ Orn. trees, $1-(1-$ $\left.\alpha)(1-\beta)), p_{2}, e\right) \wedge\left((\right.$ Orchards,$\left.\alpha), p_{2}, e\right)$. This is a more refined result, since it both keeps track of the conflict (stating that among different types of woods, orchards or ornamental trees are more plausible), and keeping a preference for the more certain information $\left((\operatorname{Orchards}, \alpha), p_{2}, e\right)$ since $\alpha>\beta$. Observe however that $1-(1-\alpha)(1-\beta))>\alpha$, which makes the statement Orchards $\vee$ Orn. trees more certain. 


\section{Summary and further research}

\subsection{Reformulation of spatial information}

In this paper, we have assumed that spatial information is expressed under the form of an attributive pair $(\langle\operatorname{property}\rangle,\langle\operatorname{parcel}(s)\rangle)$. However, it can be the case that we dispose of pieces of information expressed in a more general language built on the union of the two vocabularies $\mathscr{V}_{s}$ for the parcels and $\mathscr{V}_{i}$ for the properties. Indeed, e.g., a parcel may be described by its spatial ontology name (if any), or in extension by the disjunction of its elementary parcels, or in intention by a property formula, or more generally by a complex formula combining property and parcel vocabularies. Generally speaking, we may distinguish between four kinds of formulas with respect to their possible expression as attributive pairs :

(1) formulas expressing links between properties (which are true in each parcel). These formulas can be rewritten into classical formulas in which only literals of the vocabulary $\mathscr{V}_{i}$ appear. These rewritten formulas are i-formulas in the sense used in the beginning of this section. For example, formulas corresponding to a subsumption property expressed in the ontology $G_{i}$ are i-formulas that can be rewritten as attributive pairs. Note that a formula translating terminological exclusion between properties of an ontology $G_{i}$ may be rewritten as an attributive pair only with an "everywhere" reading. This is because such an exclusion between properties may not be true in any elementary parcel in a "somewhere" reading. More formally, an i-formula $\varphi$ expressing a property that is true for any parcel, such as terminological subsumption, can be rewritten as the pair $(\varphi, \top, e)$.

(2) formulas expressing links between parcels. These formulas can be rewritten into formulas in which only literals of the vocabulary $\mathscr{V}_{s}$ appear. The rewritten formulas are called s-formulas. The formulas corresponding to a space ontology are examples of s-formulas. A s-formula $p$ expresses links between parcels. Hence, the subsets of parcels where this link does not hold are impossible places, it means that the formula $\perp$ holds in these impossible parcels, so it can be rewritten into $(\perp, \neg p)$.

(3) formulas expressing the attribution of some properties to some geographical region. These formulas can be rewritten into attributive formulas $(\varphi, p, s)$ or $(\varphi, p, e)$ where $\varphi$ is a propositional formula representing the property to be attached to the region and $p$ is the set of elementary parcels that characterize exactly the region.

(4) there is another more complex kind of formulas that can not be rewritten into either an i-formula or an s-formula or an attributive formula. It is a formula combining the property and the parcel vocabularies (for instance, 
a formula representing the Africa countries having oil exploitations).

The fourth case is more difficult. It occurs when there is no subset of elementary parcels which exactly correspond to a property formula that describes this subset in a non ambiguous way. In other words, this problem can be handled when there exists a Galois connection allowing the translation of iformulas of interest into s-formulas and conversely. More formally, there is a Galois connection [25,3] between an i-formula and a s-formula if $(\varphi, p)$ holds together with its converse $(\neg \varphi, \neg p)$ (that could be somewhat abusively denoted as $(p, \varphi))$, i.e., all the parcels where $\varphi$ holds satisfy $p$ and all parcels in $p$ satisfy $\varphi$. In formal concept analysis [31,20] such a pair is called a concept where $\varphi$ is called the intention and $p$ the extension of this concept. More precisely, it is well known in formal concept analysis, that a relation defined on a Cartesian product of properties and objects (here, elementary parcels) gives birth to a Galois lattice where particular subsets of objects are put in a one-to-one correspondence with conjunctions of properties (that characterize these subsets).

A basic issue in spatial information handling is to be able to answer questions given a knowledge base $K$ that includes a factual base made of a set of uncertain attributive formulas and of a set of non ontology-based generic laws (concerning general attribution of properties to spatial entities). $K$ may be also augmented with ontological information expressing subsumption and mutual exclusiveness for properties and parcels. When a conclusion such as $((\varphi, \alpha), p, x)$ or $((\varphi, \alpha),[p], x)$ (where $x$ stands either for "somewhere" or for "everywhere"), or any logical combination of such formulas is obtained from $K$, it may be the case that the conclusion is not under the most suitably compact and expressive form, since $\varphi$ and $p$ may be any formulas built from $\mathscr{V}_{i}$ and $\mathscr{V}_{s}$ respectively. Clearly, reformulations can be only done by exploiting logical equivalences, such as the ones coming from Galois connections, and from ontologies. By "compact" and "expressive", we refer to different extralogical issues that amount to reformulate a logical expression. First, the idea of compacity refers to the idea of preferring the "smallest" logical expression equivalent to a given one to designate a set of parcels or even a compound property. Indeed, with respect to the ontologies $G s$ and $G i$, a conjunction may refer to a particular subclass (e.g. a set of elementary parcels corresponding to a labeled area, expressed as a disjunction, may be more compactly described by the label of this area when it exists). Expressiveness rather refers to a user preference between equivalent logical expressions (given the available knowledge) with respect to the vocabulary used in the expressions. It is in some sense a matter of focus. These reformulation issues are out of the scope of the paper. Let us note however that they are similar to the linguistic approximation problem in approximate reasoning [33], where a proper combination of linguistic labels has to be found for naming a fuzzy set approximating from above the result of a sup-min-composition-based inference. They are also rem- 
iniscent of the problem of finding a non ambiguous expression that is easy to understand for designating an object in a scene [18].

In the two following subsections, we briefly discuss other representation issues not considered in the section 4 , but which may be also of interest for spatial information.

\subsection{Percentage of a parcel}

We have already seen that one may have multiple-valued pieces of information of the form $\{(\varphi, p),(\psi, p)\}$ where $\varphi$ and $\psi$ belong to the same ontology. However, for expressing, e.g. that $p$ is both covered by orchards and cereals, we may qualitatively express that $p$ is "somewhere" covered by orchards, and "somewhere" covered by cereals. Then, the information may be augmented with the respective proportions $x$ and $y$ of the surface of $p$ corresponding to $\varphi$ and $\psi$ (Orchards and Cereals in our example). Namely, $x \%$ of the parcel is $\varphi$ and $y \%$ of the parcel is $\psi$, where $x$ and $y$ may be more or less precisely known and $x+y \leq 1$. Let us denote by $(\varphi, x \% p)$ the fact that $\varphi$ is true on at least $x \%$ of the set of elementary parcels that satisfy $p$. Then, from $(\varphi, x \% p)$ and $\left(\varphi, y \% p^{\prime}\right)$, one can clearly deduce $\left(\varphi, \frac{x \% \cdot s(p)+y \% . s\left(p^{\prime}\right)}{s(p)+s\left(p^{\prime}\right)} \% \quad p \vee p^{\prime}\right)$ provided that $p \wedge p^{\prime} \equiv \perp$, where $s(p)$ denotes the surface of parcel $p$. However, it is worth noticing that from $(\varphi, x \% p)$ and $(\psi, y \% p)$, one cannot deduce $(\varphi \vee \psi,(x+y) \% p)$ in general, except if one has the further information that $\varphi$ and $\psi$ are mutually exclusive (then one should have $x+y \leq 1$ for consistency reason). Clearly also, from $(\varphi, x \% p)$ and $\left(\varphi, y \% p^{\prime}\right)$ one cannot deduce anything on $p \wedge p^{\prime}$ without knowing what percentages of $p$ and of $p^{\prime}, p \wedge p^{\prime}$ represents.

\subsection{Positive vs. negative information}

Generally speaking, there are two ways of interpreting incomplete information (should it be numerical or symbolic).

- Information can be interpreted "negatively". Namely, any interpretation that is not compatible with the stated pieces of information is judged to be impossible. This kind of information, which is the one usually needed, can be termed as "exhaustively closed". For instance, if one states that there is some part of a parcel $p$ that should be "cereals or meadows", one generally means that any other thing is more or less excluded, and this is encoded by a necessity measure-based possibilistic logic constraint, namely $(($ Cereals $\vee$ Meadows,$\alpha), p)$, where $\alpha$ is the certainty level associated with the piece of information "cereals or meadows". In terms of a possibility 
distribution $\pi$, this means that the plausibility $\pi(\omega)$ of an interpretation $\omega$ for parcel $p$ is such that:

$$
\pi(\omega)= \begin{cases}1 & \text { if } \omega \models \text { Cereals } \vee \text { Meadows } \\ 1-\alpha & \text { if } \omega \models \neg \text { Cereals } \wedge \neg \text { Meadows }\end{cases}
$$

In case "cereals" and "meadows" are perceived as mutually exclusive, $\pi(\omega)$ will be modified by enforcing $\pi(\omega)=0$ if $\omega \models$ Cereals $\wedge$ Meadows, i.e., by adding ( $\neg$ Cereals $\vee \neg$ Meadows, 1 ) to the knowledge base.

- However, there exists also a positive type of understanding [14] that focuses on what is possible for sure (because it has been observed), and does not refer to what is known to be impossible. This kind of information can be viewed as an "open" set of values, the piece of information "cereals or meadows" then means that what has been observed in $p$ could be meadows or cereals, with the implicit assumption that it might be other things. Then another type of distribution $\delta$ should be used, where

$$
\delta(\omega)= \begin{cases}1 & \text { if } \omega \models \text { Meadows } \\ 1 & \text { if } \omega \models \text { Cereals } \\ 0 & \text { otherwise }\end{cases}
$$

Now, $\delta(\omega)=0$ does not mean (as $\pi(\omega)$ does) that $\omega$ is impossible for parcel $p$, but just that we have no clue at all for suggesting that $\omega$ is possible for sure. Note that when the clues guaranteeing possibility are not fully strong then $\delta(\omega)$ becomes graded between 0 and 1. This second type of information can also be handled at the syntactic level in a new form of possibilistic logic [4], which could be also "spatialized" in order to have attributive formulas corresponding to this new type.

\section{Conclusion}

After having identified representational needs (references to ontologies, uncertainty) when dealing with spatial information, a general logical setting has been proposed. This setting offers a non-ambiguous representation, propagates uncertainty in a possibilistic manner, and provides also the basis for handling multiple source information fusion. We have chosen to use a possibilistic rather a probabilistic modeling of uncertainty for several reason. First the possibilistic representation is qualitative, which seems reasonable when information is poor. Second the possibilistic setting is particularly suitable for the representation of states of partial or complete ignorance. Third, the logical handling of possibilistic uncertainty is particularly simple with possibilistic logic. 
The problem of the approximation of pieces of information expressed in a given vocabulary into another related vocabulary has been mentioned in several places in the paper. This reformulation problem is clearly a line for further research and a concern that seems closely related to the idea of rough set approximations [26,29], and also to the exploitation of Galois correspondences for moving from extensional to intentional descriptions and conversely. Another issue of interest would be to allow for uncertain or default inheritance in ontologies. Note that, since subsumption relations can be easily added to the pieces of attributive spatial information, it would be possible to make some of these relations uncertain in our framework.

As discussed along the paper, the handling of spatial information raises general problems encountered with other types of information such as the representation of uncertainty or the use of the closed world assumption, as well as specific problems. Particular representation issues regarding spatial information are related to the need of "localizing" properties. First, this requires the use of two vocabularies referring respectively to parcels and to properties. Moreover, we have seen that it is often important to explicitly distinguish between the cases where a property holds everywhere or somewhere into a parcel. Concerning the fusion of information, it is necessary to merge pieces of information relative to the same area. This requires to be able to "project" the available information on a considered area. Moreover, it is also necessary to handle the "somewhere" and "everywhere" information in the fusion process.

Another important issue, not considered in the paper, is the conjoint use of pieces of information pertaining to different parcels together with dependency relations (induced by neighboring properties for instance) between these parcels when merging information. A similar problem is encountered in updating, where the situation at time $t+1$ generally depends on situation at time $t$. When the information about the $a_{i}(p)$ 's is represented by possibility distributions, we need to apply the extension principle to $f$ or $R$, or its syntactic counterpart when dealing with symbolic labels, see [5], for evaluating $a(p)$.

\section{Acknowledgments}

This work takes place in the framework of an Inter-Regional Action Project $n$ 05013992 "GEOFUSE: Fusion d'informations géographiques incertaines", partially supported by the Conseils Régionaux of Midi-Pyrénées and of ProvenceAlpes-Côte d'Azur. This work has also benefited from several discussions with Robert Jeansoulin and Odile Papini. 


\section{References}

[1] F. Baader, D. Calvanese, D.L. McGuinness, D. Nardi, and P.F. Patel-Schneider (eds). The Description Logic Handbook. Cambridge University Press, 2002.

[2] S. Balley, C. Parent, and S. Spaccapietra. Modelling geographic data with multiple representations. International Journal of Geographical Information Science, 18(4):327 - 352, June 2004.

[3] M. Barbut and B. Monjardet. Ordre et Classification: Algèbre et combinatoire. Hachette, 1970.

[4] S. Benferhat, D. Dubois, S. Kaci, and H. Prade. Bipolar possibility theory in preference modeling: Representation, fusion and optimal solutions. Information Fusion, 7:135-150, 2006.

[5] S. Benferhat, D. Dubois, and H. Prade. Kalman-like filtering and updating in a possibilistic setting. In Proc.14th Europ.Conf.on Artificial Intelligence (ECAI 2000), Berlin, pages 8-12. IOS Press, 20-25 aot 2000.

[6] S. Benferhat, D. Dubois, and H. Prade. A computational model for belief change and fusing ordered belief bases. In Mary-Anne Williams and Hans Rott, editors, Frontiers in Belief Revision, pages 109-134. Kluwer Academic Publishers, 2001.

[7] I. Bloch and A. Hunter (Eds). Fusion: General Concepts and Characteristics. International Journal of Intelligent Systems, 16(10):1107-1134, oct 2001.

[8] E. Caianiello. C-calculus: an overview. In E.R. Caianiello and M.A. Aizerman, editors, Topics in the General Theory of Structures, pages 163-173. D. Reidel Dordrecht, 1987.

[9] E Caianiello and A. Ventre. A model for C-calculus. International journal of General Systems, 11:153-161, 1985.

[10] F. Dupin de Saint Cyr and H. Prade. Multiple-source data fusion problems in spatial information systems. In Proc. of the 11th International Conference on Information Processing and Management of Uncertainty in Knowledge-Based Systems (IPMU'06), pages 2189-2196, Paris, France, 02/07/06-07/07/06 2006.

[11] O. Doukari and R. Jeansoulin. Space contained conflict revision to allow consistency checking of spatial decision support. In AGILE $200^{7}$ Conference, Aalborg, DK, May 2007.

[12] D. Dubois, J. Lang, and H. Prade. Possibilistic logic. In D.M. Gabbay, C.J. Hogger, and J.A. Robinson, editors, Handbook of logic in Artificial Intelligence and logic programming, volume 3, pages 439-513. Clarendon Press - Oxford, 1994.

[13] D. Dubois and H. Prade. Possibility Theory. Plenum Press, 1988. 
[14] D. Dubois and H. Prade. A bipolar possibilistic representation of knowledge and preferences and its applications. Fuzzy Logic and Applications (Revised Selected Papers From the Inter. Workshop WILF'05, Crema, Italy, September 2005), LNAI 3849:1-10, 2006.

[15] D. Dubois and H. Prade. Toward a multiple-agent extension of possibilistic logic. In Proc. IEEE International Conference on Fuzzy Systems (FUZZIEEE2007), London, UK, 23-26 July 2007.

[16] D. Dubois, H. Prade, and R. Yager. Merging fuzzy information. In H. Prade J. Bezdek, D. Dubois, editor, Fuzzy Sets in Approximate Reasoning and Information Systems, pages 335-401. Kluwer, Boston, the handbooks of fuzzy sets series edition, 1999.

[17] M. Duckham and M. Worboys. An algebraic approach to automated information fusion. International Journal of Geographic Information Systems, 19(5):537$557,2005$.

[18] H. Farreny and H. Prade. On the best way of designating objects in sentence generation. Kybernetes, 13(1):43-46, 1984.

[19] F. Fonseca, M. Egenhofer, P. Agouris, and G. Cmara. Using ontologies for integrated geographic information systems. Transactions in GIS, 6(3):231-257, 2002 .

[20] B. Ganter and R. Wille. Formal Concept Analysis, Mathematical Foundations. Springer-Verlag, 1999.

[21] M. Goodchild and R. Jeansoulin. Data Quality in Geographic Information : from Error to Uncertainty. Hermés, Paris, 1998. 192 pages.

[22] M.F. Goodchild, M. Yuan, and T.J. Cova. Towards a general theory of geographic representation in gis. International Journal of Geographical Information Science, 21(3):239-260, 2007.

[23] R. Jeansoulin, T.T. Pham, and V. Phan-Luong. A quality-aware theme fusion for spatial information. In International. Conference on Formal Concept Analysis, FCA200\%, Clermont-Ferrand, France, 12-16 fvrier 2007.

[24] A.-M. Olteanu. A multi-criteria fusion approach for geographical data matching. In Proceedings of the $5^{\text {th }}$ International Symposium on Spatial Data Quality (ISSDQ'07), June 2007.

[25] O. Ore. Galois connexions. Transactions of the American Mathematical Society, 55:493-513, 1944.

[26] Z. Pawlak. Rough sets: Theoretical aspects of reasoning about data. System theory, knowledge engineering and problem solving, 9, 1991.

[27] F. Petry, M. Cobb, L. Wen, and H. Yang. Design of system for managing fuzzy relationships for integration of spatial data in querying. Fuzzy Sets and Systems, 140(1):51-73, November 2003. 
[28] T. Trung Pham. Fusion de l'information géographique hiérarchisée. PhD thesis, Université de Provence, septembre 2005.

[29] A. Skowron, R. Swiniarski, and P. Synak. Approximation spaces and information granulation. Lecture Notes in Computer Science, 3400:175 - 189, Jan 2005.

[30] Steffen Staab and Rudi Studer (eds). Handbook on Ontologies. Springer, 2004.

[31] R. Wille. Restructuring lattice theory: an approach based on hierarchies of concepts. In I. Rival, editor, Ordered Sets, pages 445-470. D. Reidel, DordrechtBoston, 1982.

[32] E. Wurbel, O. Papini, and R. Jeansoulin. Revision: an application in the framework of GIS. In proc. of the 7th International Conference on Principles of Knowledge Representation and Reasoning, KR'2000, pages 505516, Breckenridge, Colorado, USA, avril 2000.

[33] L. Zadeh. A theory of approximate reasoning. Machine Intelligence, 9:149-194, 1979. 